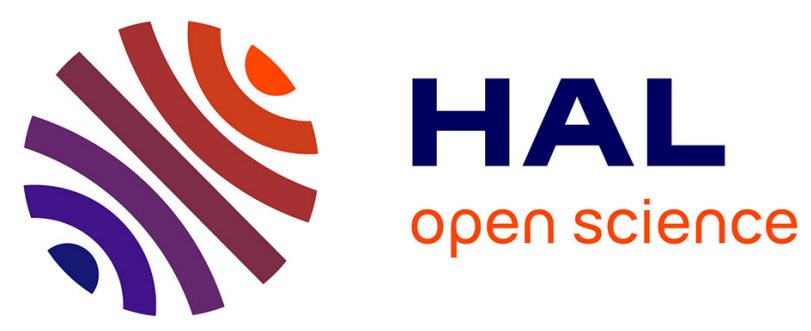

\title{
The low-lying excited states of neutral polyacenes and their radical cations: a quantum chemical study employing the algebraic diagrammatic construction scheme of second order
}

Stefan Knippenberg, Jan-Hendrik Starcke, Michael Wormit, Andreas Dreuw

\section{To cite this version:}

Stefan Knippenberg, Jan-Hendrik Starcke, Michael Wormit, Andreas Dreuw. The low-lying excited states of neutral polyacenes and their radical cations: a quantum chemical study employing the algebraic diagrammatic construction scheme of second order. Molecular Physics, 2010, 108 (19-20), pp.2801-2813. 10.1080/00268976.2010.526643 . hal-00637800

\section{HAL Id: hal-00637800 https://hal.science/hal-00637800}

Submitted on 3 Nov 2011

HAL is a multi-disciplinary open access archive for the deposit and dissemination of scientific research documents, whether they are published or not. The documents may come from teaching and research institutions in France or abroad, or from public or private research centers.
L'archive ouverte pluridisciplinaire HAL, est destinée au dépôt et à la diffusion de documents scientifiques de niveau recherche, publiés ou non, émanant des établissements d'enseignement et de recherche français ou étrangers, des laboratoires publics ou privés. 


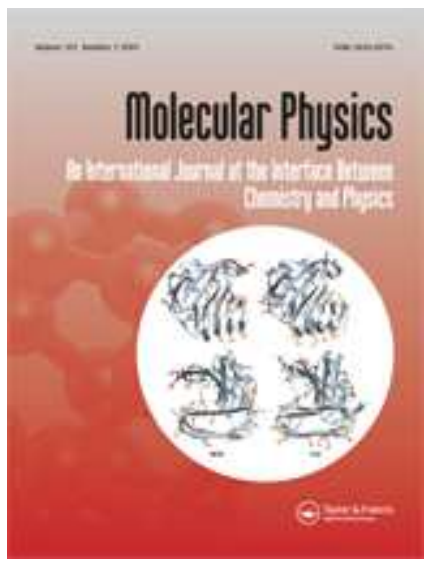

\section{The low-lying excited states of neutral polyacenes and their radical cations: a quantum chemical study employing the algebraic diagrammatic construction scheme of second order}

\begin{tabular}{|c|c|}
\hline Journal: & Molecular Physics \\
\hline Manuscript ID: & TMPH-2010-0370 \\
\hline Manuscript Type: & Special Issue Paper - MQM 2010 \\
\hline $\begin{array}{r}\text { Date Submitted by the } \\
\text { Author: }\end{array}$ & 16-Sep-2010 \\
\hline Complete List of Authors: & $\begin{array}{l}\text { Knippenberg, Stefan; University of Frankfurt, Institute of Physical } \\
\text { and Theoretical Chemistry } \\
\text { Starcke, Jan-Hendrik; University of Frankfurt, Institute of Physical } \\
\text { and Theoretical Chemistry } \\
\text { Wormit, Michael; University of Frankfurt, Institute of Physical and } \\
\text { Theoretical Chemistry } \\
\text { Dreuw, Andreas; University of Frankfurt, Institute of Physical and } \\
\text { Theoretical Chemistry }\end{array}$ \\
\hline Keywords: & $\begin{array}{l}\text { electron propagator, algebraic diagrammatic construction, excited } \\
\text { states, polyacenes, radical cations }\end{array}$ \\
\hline \multicolumn{2}{|c|}{$\begin{array}{l}\text { Note: The following files were submitted by the author for peer review, but cannot be converted } \\
\text { to PDF. You must view these files (e.g. movies) online. }\end{array}$} \\
\hline Submit.zip & \\
\hline
\end{tabular}




1
1
3
4
5
6
7
8
9
10
11
12
13
14
15
16
17
18
19
20
21
22
23
24
25
26
27
28
29
30
31
32
33
34
35
36
37
38
39
40
41
42
43
44
55
50
56
57
48
59
50
51
53
50

\section{SCHOLARONE ${ }^{m}$ \\ Manuscripts}

7 


\title{
RESEARCH ARTICLE
}

\author{
The low-lying excited states of neutral polyacenes and their \\ radical cations: a quantum chemical study employing the \\ algebraic diagrammatic construction scheme of second order
}

\author{
Stefan Knippenberg, Jan Hendrik Starcke, Michael Wormit and Andreas Dreuw* \\ Institut für Physikalische und Theoretische Chemie, Goethe-Universität Frankfurt, Max \\ von Laue-Str. 7, 60438 Frankfurt, Germany \\ (Received 00 Month 200x; final version received 00 Month 200x)
}

\begin{abstract}
The vertical excited electronic states of linearly fused neutral polyacenes and their radical cations have been investigated using the algebraic diagrammatic construction scheme of second order $(\mathrm{ADC}(2))$. While strict $\mathrm{ADC}(2)(\mathrm{ADC}(2)-\mathrm{s})$ correctly reproduces trends for mainly singly excited states, in extended $\operatorname{ADC}(2)(\operatorname{ADC}(2)-x)$ the description of doubly excited states is critically improved. It is shown that a combined application of strict and extended ADC(2) nicely reveals the importance of doubly excited configurations in the description of the excited state spectrum of the neutral polyacenes. In contrast to general expectation, our calculations of the lowest excited states of the radical cations of the polyacenes employing unrestricted $\mathrm{ADC}(2)$-s and $\mathrm{ADC}(2)$-x identified one $\mathrm{B}_{1 g}$ electronic transition whose excitation energy increases with increasing molecular size. Thorough analysis of this electronic state and the involved molecular orbitals relates this unusual phenomenon to an increasing energy gap between the doubly occupied molecular orbital, out of which an electron is excited, and the singly occupied orbital, into which it is excited.
\end{abstract}

Keywords: electron propagator, algebraic diagrammatic construction, excited states, polyacenes, radical cations

\section{Introduction}

The development of efficient quantum chemical methods for the accurate calculation of excited state properties of large molecular systems is one of the greatest challenges of modern theoretical chemistry $[1,2]$. Today, several excited state methods are already available for the investigation of large molecules, among which time-dependent density functional theory (TDDFT) [3-5] is by far the most widely used. Complete active-space self-consistent field (CASSCF) methods [6, 7], symmetry-adapted cluster configuration interaction (SAC-CI) [8] and also approximate linear-response coupled-cluster theory of second order (RI-CC2) [9-12] are powerful wavefunction-based approaches. Nevertheless, all existing methods possess individual weaknesses and drawbacks $[1,2]$. The success of the available methods is manifested by ample excellent applications in the fields of photochemistry and photobiology [13-24].

Even more than for closed-shell molecules, theoretical methods are urgently needed to reliably provide information about excited states of open-shell molecules, since neutral and charged radicals are of crucial importance in a broad range of chemical and biological processes. Unrestricted formulations and implementations

*Corresponding author. Email: andreas.dreuw@theochem.uni-frankfurt.de 
a)<smiles>c1ccc2ccccc2c1</smiles>

b)<smiles>c1ccc2cc3ccccc3cc2c1</smiles>

c)<smiles>c1ccc2cc3cc4ccccc4cc3cc2c1</smiles>

d)<smiles>c1ccc2cc3cc4cc5ccccc5cc4cc3cc2c1</smiles>

e)

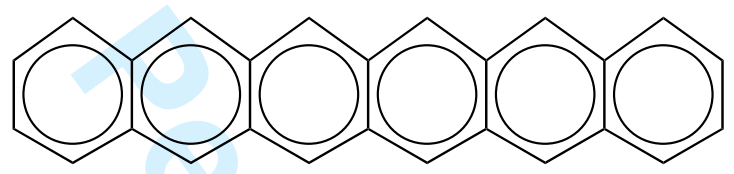

Figure 1. Molecular structure of the linearly fused polyacenes from a) naphthalene to b) anthracene, c) tetracence, d) pentacene and e) hexacene.

of coupled cluster (CC) methods like equation-of-motion CC (EOM-CC) [25, 26] and linear response CC (LR-CC) [27-29] provide good results for excited states of smaller open-shell molecules, while approximate CC methods like CC2 [10, 12] and CASSCF theories [30-32] are available for medium-sized radicals, too. Of course, TDDFT can also be employed to compute electronic spectra of radicals. However, here one certainly faces all problems known from closed-shell systems [5].

An elegant, however, until today mostly overseen method for the calculation of excited states is the algebraic diagrammatic construction (ADC) scheme of the polarization propagator [33-37], originally derived in the realm of Green's function theory $[38,39]$. The recent development of efficient computer codes for ADC is a prerequisite for its routine application to molecular systems of chemical and biophysical interest [40-43]. Although the ADC scheme is general and can be expanded to arbitrarily high-orders in perturbation theory, only second order ADC $(\mathrm{ADC}(2))$ is currently computationally efficient enough to be applicable to large and medium-sized molecules on present-day computers. Recently, the $\mathrm{ADC}(2)-\mathrm{x}$ scheme has been extended to an unrestricted spin-orbital formalism allowing for the calculation of excited states of open-shell radicals [42, 43]. For energetically lowlying states of medium-sized open-shell doublet radicals, the UADC(2)-x scheme turned out to yield excitation energies with an overall accuracy of about $0.3 \mathrm{eV}$ [42].

In this work, ADC and UADC schemes are employed to investigate the vertical excited states of neutral, linearly fused polyacenes (PAC) and their radical cations. In figure 1, the series from naphthalene with two fused rings up to hexacene with six rings is displayed. Polyacenes belong to the class of polycyclic aromatic hydrocarbons (PAHs) which form a large group of conjugated $\pi$-electron systems of fundamental importance in many research areas of chemistry as well as in astro- 
physics and materials science [44-46].

Polyacenes pose a challenge to quantum chemistry, because the extended $\pi$ electron conjugation leads to long-range electron correlations, which must be taken into account for a meaningful description of the electronic structure and, thus, of many physical and chemical properties (see for example [47-53]). Therefore, the linear PACs and their radical cations represent excellent test sets to explore the applicability and the limits of the restricted and unrestricted $\mathrm{ADC}(2)$ methods.

In this work, we focus on the energetically low-lying excited electronic states of the neutral polyacenes naphthalene up to hexacene and their radical cations in the near UV and visible range of the optical spectrum. In particular, the influence of doubly excited states in that energy regime lies at the heart of our study, which will be discussed in detail below. On one hand the accuracy of the $\operatorname{ADC}(2)$ schemes and its applicability to these highly correlated class of conjugated systems shall be investigated. On the other hand, some more light is to be shed on the excited states and their properties, in particular on the ones of the radical cations, which have only been scarcely studied theoretically so far.

The paper is organized as follows. For the paper to be self-contained and since $\mathrm{ADC}$ is a rather uncommon approach, we briefly review the derivation of the $\operatorname{ADC}(2)$ schemes in Section 2, where also a brief comparison to other excited states methods is made. After presenting the computational details, first the low-lying excited states of neutral polyacenes will be studied and compared to existing literature values in section 4 . Then, we will focus onto the excited states of the corresponding radical cations (Section 5) The paper concludes with a brief summary of the major results and an outlook (Section 6).

\section{Theory}

Algebraic expressions of the ADC scheme for excited states have first been derived for closed-shell molecules employing diagrammatic perturbation theory of the polarization propagator using the typical Møller-Plesset partition of the Hamilton operator $[33-35,54]$. From that point of view, the choice of the otherwise unusual name becomes obvious. However, in addition, the ADC equations can be derived in an elegant way employing the intermediate state representation (ISR) [37], which will be presented in the following in a little bit more detail. Besides an elegant derivation, the ISR also provides access to the wavefunctions of the excited states and thus to the computation of excited state properties.

The starting point of the derivation of the ADC scheme via ISR is the exact N-electron ground state wavefunction $\left|\Psi_{0}^{N}\right\rangle$, to which physical excitation operators are applied to generate a "correlated" set of excited state wavefunctions $\left|\Psi_{J}^{0}\right\rangle$

$$
\left|\Psi_{J}^{0}\right\rangle=\hat{C}_{J}\left|\Psi_{0}^{N}\right\rangle
$$

with

$$
\left\{\hat{C}_{J}\right\}=\left\{c_{a}^{\dagger} c_{k} ; c_{a}^{\dagger} c_{k} c_{b}^{\dagger} c_{l}, a<b, k<l ; \ldots\right\}
$$

where the operators $c_{p}^{\dagger}\left(c_{p}\right)$ of second quantization are associated with one-particle states $\left|\phi_{p}\right\rangle$ of the Hartree-Fock ground state $\left|\Phi_{0}^{N}\right\rangle$. As usual, the subscripts $a, b, c, \ldots, i, j, k, \ldots, p, q, r, \cdots$ label unoccupied (virtual), occupied and general orbitals, respectively. The excitation operators $\hat{C}_{J}$ naturally decompose into classes depending on the number of electrons that are excited. 
Although these "correlated" excited states form a complete set of N-electron states provided that $\left\langle\Psi_{0}^{N} \mid \Phi_{0}^{N}\right\rangle \neq 0[55,56]$, they are not orthonormal. However, it is always possible to construct an orthonormal basis by successive Gram-Schmidt orthogonalization of the excitation classes starting from the exact ground state, the singly excited states, doubly excited states, etc. eventually leading to the so-called intermediate states (IS) $\left|\tilde{\Psi}_{J}\right\rangle[57,58]$. Finally, it is obvious that the exact excited states $\left|\Psi_{n}\right\rangle$ can be represented in this IS basis via

$$
\left|\Psi_{n}\right\rangle=\sum_{J} X_{n J}\left|\tilde{\Psi}_{J}\right\rangle
$$

To arrive at algebraic expressions to compute excitation energies, one represents the shifted Hamiltonian $\hat{H}-E_{0}$ in the IS basis to obtain

$$
\left\langle\tilde{\Psi}_{I}\left|\hat{H}-E_{0}^{N}\right| \tilde{\Psi}_{J}\right\rangle=\left\langle\Psi_{0}^{N}\left|\tilde{C}_{I}^{\dagger}\left[\hat{H}, \tilde{C}_{J}\right]\right| \Psi_{0}^{N}\right\rangle=M_{I J} .
$$

Diagonalization of the hermitian ADC matrix $\mathbf{M}$, i.e. solution of the corresponding ISR secular equation,

$$
\mathbf{M X}=\mathbf{X} \boldsymbol{\Omega}, \quad \mathbf{X}^{\dagger} \mathbf{X}=1
$$

yields as eigenvalues the excitation energies,

$$
\Omega_{n}=E_{n}-E_{0}
$$

and as eigenvectors the coefficients of the exact excited states in the IS expansion (eq. 3). In general, the transition dipole moments

$$
T_{n}=<\Psi_{n}|\hat{\mu}| \Psi_{0}>
$$

where $\hat{\mu}$ is the typical dipole operator, are given by

$$
T_{n}=\mathbf{X}_{\mathbf{n}}^{\dagger} \mathbf{F}(\mu)
$$

as the scalar products of the eigenvectors $\mathbf{X}_{n}$ with the so-called effective transition moments $\mathbf{F}(\mu)$. The corresponding oscillator strength is obtained according to the standard expression

$$
f_{n}=\frac{2}{3} \Omega_{n}<T_{n}>^{2} .
$$

Note that the derivation started from the exact correlated ground state wavefunction yielding a full-ADC scheme providing exact excitation energies and transition moments. However, the exact ground state is of course not known, and thus one has to resort to a suitable approximation as starting point and to derive the corresponding ISR matrix elements. In fact, the level of approximation of the ground state wave function determines the order of the ADC scheme. For example, when an MP2 ground state is chosen, the corresponding ISR derivation yields $\mathrm{ADC}(2)$, MP3 results in $\mathrm{ADC}(3)$, etc. The algebraic expressions of the matrix elements $M_{I J}$ (eq. (4)) are typical perturbation theoretical expressions, which have been derived in detail for $\mathrm{ADC}(2)$ and $\mathrm{ADC}(3)$ in Refs. [34-36]. Since the ADC(n) excitation energies are computed with respect to the corresponding MPn ground state, $\mathrm{ADC}(2)$, 
for instance, should only be applied to molecular systems whose ground state is well described by a single determinant such that MP2 is a reasonable approximation.

The extension of the ISR formalism to a spin-orbital basis is straightforward and enables the derivation of unrestricted ADC schemes [42]. These UADC schemes can be employed to compute excited states of open-shell radicals. The major changes in the derivation occur in the choice of the excitation operators in equation (2), where now attention has to be paid that successive annihilation and creation operators act on spin-orbitals with the same spin, thereby excluding so-called spin-flip excitations in which the spin state of the excited electron is changed. Furthermore, UMP2 must be chosen as ground state reference to eventually derive UADC (2).

A strict and an extended variant of $\operatorname{ADC}(2)$ have been developed [34], and are further referred to as $\mathrm{ADC}(2)-\mathrm{s}$ and $\mathrm{ADC}(2)-\mathrm{x}$, respectively. While in the former scheme couplings between doubly excited configurations are not contained in the $2 p 2 h / 2 p 2 h$ block of the ADC matrix, i.e. the $2 p 2 h / 2 p 2 h$-block of $\mathbf{M}$ is diagonal, they are included to first order in an ad hoc fashion in $\operatorname{ADC}(2)-\mathrm{x}$. Originally these first-order coupling terms stem from the higher-order $\mathrm{ADC}(3)$ scheme. Nevertheless, their inclusion leads to an overall improved treatment of excited states with double excitation character in the ADC(2)-x scheme. Typically, excited states with significant double excitation character are energetically too high in ADC(2)-s, and their energy decreases substantially relative to states with mostly single excitation character when $\mathrm{ADC}(2)-\mathrm{x}$ is used. Thus, both schemes can be used in comparison to detect appearance and importance of low-lying doubly excited states, as has been demonstrated previously for the $2 A_{g}^{-}$state of linear polyenes [41].

From a numerical point of view, ADC combines perturbation theory with configuration interaction (CI). It is size-consistent and, in addition, the ADC matrix $\mathbf{M}$ is hermitian. Moreover, ADC is more compact than CI or CC. Compactness is generally defined as the size of the configuration space required to describe ground and singly excited states consistently through a particular order of perturbation theory. While CI methods generally require the full configuration space, i.e. for a consistent treatment of these primary states to say $n$th order of perturbation theory one needs all $n$-tuply excited configurations. When the order of perturbation theory is even, say $2 m$, both $\mathrm{ADC}$ and $\mathrm{CC}$ are identically compact and require a configuration space of $m+1$. For odd orders of perturbation theory, $2 m+1$, ADC is slightly more compact with a configuration space size of also $m+1$ compared to $m+2$ for the CC methods. For a detailed comparison of ADC, CC and CI the reader is referred to $[54,59,60]$.

\section{Computational Details}

The geometrical parameters of the neutral polyacenes and their radical cations have been optimized employing second order Møller-Plesset perturbation theory (MP2) together with Pople's 6-31G* basis set. For the open-shell radical cations an unrestricted Hartree-Fock reference (UHF) has been employed throughout all reported calculations. The vertical excited states have been calculated using the strict and extended ADC scheme of second order, $\operatorname{ADC}(2)-\mathrm{s}$ and $\mathrm{ADC}(2)$-x, respectively, which have been implemented into a development version of the QCHEM-3.2 quantum chemistry program package [61]. For comparison, the $\mathrm{B}_{2 u}$ and $\mathrm{B}_{3 u}$ excited states of the neutral compounds have been computed also with the equationof-motion coupled cluster singles and doubles (EOM-CCSD) method [29, 62]. Throughout the calculations the core electrons have been frozen, and the $\mathrm{D}_{2 h}$ symmetry point group has been exploited. The vertical excited states of the polyacene radical cations have also been calculated using unrestricted TDDFT in combina- 
tion with the hybrid Becke three-parameter Lee-Yang-Parr (B3LYP) xc-functional and the $6-31 \mathrm{G}^{*}$ basis set $[3-5]$.

\section{Excited State Properties of Neutral Polyacenes}

Neutral linearly fused polyacenes possess $\mathrm{D}_{2 h}$ symmetry, and a closed-shell $\mathrm{A}_{1 g}$ electronic ground state. They are well-known to possess two prominent excited states in the low energy region dominating the experimental absorption spectrum: a short-axis polarized, so-called $\mathrm{L}_{a}\left(\mathrm{~B}_{2 u}\right)$ state and a long-axis polarized $\mathrm{L}_{b}\left(\mathrm{~B}_{3 u}\right)$ state. The existence of other dark excited states in this energy region has been indicated by early two-photon absorption spectra of naphthalene, in which an excited state with gerade symmetry has been observed around $5.5 \mathrm{eV}$ [63-65]. More than thirty years ago, Tavan and Schulten computed the excited states of linearly fused polyacenes from naphthalene up to pentacene using a configuration interaction doubles (D-CI) approach based on the semi-empirical Pariser-Parr-Pople (PPP) Hamiltonian [66]. Already then, they noticed the importance of double excitations for a reliable treatment of the low-lying excited states of these molecules. "New" doubly excited states with gerade-symmetry were observed, and indeed $\mathrm{A}_{g}$ was found to drop energetically below the optically allowed $B_{3 u}$ state when the number of fused benzene rings reaches five, i.e. pentacene. In addition, the $\mathrm{B}_{2 u}\left(\mathrm{~L}_{a}\right)$ and $\mathrm{B}_{3 u}$ $\left(\mathrm{L}_{b}\right)$ states were found to change order from naphthalene to tetracene, the former becoming lower in energy than the latter. In a recent study, the excited electronic states of these neutral polyacenes have been re-investigated by Marian and Gilka using a semi-empirical DFT/MRCI approach [67]. Their results agree largely with the early calculations of Tavan and Schulten. The low-lying doubly excited $\mathrm{A}_{g}$ and $\mathrm{B}_{1 g}$ states have been confirmed, and the $\mathrm{A}_{g}$ state has been shown to become even the lowest excited state from hexacene on.

Recently, Parac and Grimme pointed out that TDDFT yields substantial errors even in the description of the $\mathrm{B}_{2 u}\left(\mathrm{~L}_{a}\right)$ state while its counterpart $\mathrm{B}_{3 u}\left(\mathrm{~L}_{b}\right)$ is described reasonably well $[68,69]$. CC2, on the other hand provides a balanced description of these two states in good agreement with the experiment [68]. Since both the CC2 method and TDDFT are not capable of describing doubly excited states accurately, one can exclude these TDDFT problems to arise from double excitation character, as it is for instance the case for the $2 \mathrm{~A}_{g}^{-}$state of linear polyenes [41] or as it would be for the $\mathrm{A}_{g}$ and $\mathrm{B}_{1 g}$ states of the polyacenes as we will see later.

All reported studies including also excited states of the polyacenes with gerade symmetry rely on multi-reference treatments based on some semi-empirical model Hamiltonian. Note that also the DFT/MRCI approach used by Marian and Gilka and devised by Grimme also contains semi-empirical parameters to screen the Coulomb integrals [16]. Here, on the other hand, ab initio single-reference ADC(2) as well as EOM-CCSD approaches have been used to calculate the vertical excited states of the neutral linearly fused polyacenes from naphthalene up to hexacene, and the obtained results for the lowest $1^{1} \mathrm{~B}_{3 u}\left(\mathrm{~L}_{b}\right), 1^{1} \mathrm{~B}_{2 u}\left(\mathrm{~L}_{a}\right), 2^{1} \mathrm{~A}_{g}$ and $1^{1} \mathrm{~B}_{1 g}$ states are compiled in Table 1.

For naphthalene the $1^{1} \mathrm{~B}_{3 u}\left(L_{b}\right)$ state corresponds to the lowest excited $\mathrm{S}_{1}$ state at all employed levels of theory supported by the known experimental excitation energy. This state is well described as a single excitation, and can be represented as linear combination of HOMO- $\rightarrow$ LUMO and $\mathrm{HOMO} \rightarrow \mathrm{LUMO}+1$ excited determinants. While ADC(2)-s, EOM-CCSD, TDDFT/B3LYP and CC2 yield very similar values, they all overestimate the vertical excitation energy by about $0.4-0.5 \mathrm{eV}$, and only DFT/MRCI gives an excitation energy of $4.15 \mathrm{eV}$ in remarkable agreement 
Table 1. Computed vertical excitation energies $(\mathrm{eV})$ and oscillator strengths (in parenthesis) of the four lowest excited states of the neutral polyacenes naphthalene (2) to hexacene (6) at ADC(2)-s, ADC(2)-x and EOM-CCSD level of theory compared with literature data at DFT/MRCI, TDDFT and CC2 level and experimental values.

\begin{tabular}{|c|c|c|c|c|c|c|c|}
\hline $\mathrm{n}$ & $\operatorname{ADC}(2)-\mathrm{s}$ & $\operatorname{ADC}(2)-\mathrm{x}$ & EOM-CCSD & $\mathrm{DFT} / \mathrm{MRCI}^{\mathrm{a}}$ & TDDFT/B3LYP & $\mathrm{CC} 2^{\mathrm{b}}$ & Expt. ${ }^{c}$ \\
\hline \multicolumn{8}{|c|}{$\mathbf{1}^{1} \mathbf{B}_{3 u}\left(\mathbf{L}_{b}\right)$} \\
\hline 2 & $4.57(0.000)$ & $3.51(0.000)$ & 4.47 & 4.15 & 4.47 & 4.46 & 4.13 \\
\hline 3 & $4.00(0.001)$ & $3.01(0.001)$ & 3.94 & 3.59 & 3.87 & 3.89 & 3.64 \\
\hline 4 & $3.61(0.002)$ & $2.67(0.002)$ & 3.58 & 3.22 & 3.47 & 3.52 & 3.39 \\
\hline 5 & $3.36(0.000)$ & $2.42(0.000)$ & & 2.99 & 3.21 & 3.27 & 3.12 \\
\hline 6 & $3.18(0.000)$ & $2.28(0.000)$ & & 2.76 & 3.02 & 3.09 & 2.87 \\
\hline \multicolumn{8}{|c|}{$\mathbf{1}^{1} \mathbf{B}_{2 u}\left(\mathbf{L}_{a}\right)$} \\
\hline 2 & $5.09(0.104)$ & $4.47(0.098)$ & 5.37 & 4.66 & 4.38 & 4.88 & 4.66 \\
\hline 3 & $3.87(0.109)$ & $3.27(0.092)$ & 4.22 & 3.51 & 3.21 & 3.69 & 3.60 \\
\hline 4 & $3.04(0.102)$ & $2.46(0.081)$ & 3.44 & 2.74 & 2.43 & 2.90 & 2.88 \\
\hline 5 & $2.46(0.000)$ & $1.90(0.000)$ & & 2.22 & 1.89 & 2.35 & 2.37 \\
\hline 6 & $2.05(0.000)$ & $1.49(0.000)$ & & 1.85 & 1.49 & 1.95 & 2.01 \\
\hline \multicolumn{8}{|c|}{$\mathbf{2}^{1} \mathbf{A}_{g}$} \\
\hline 2 & $6.44(0.000)$ & $5.04(0.000)$ & 6.39 & 5.73 & & & \\
\hline 3 & $5.78(0.000)$ & $3.79(0.000)$ & 5.76 & 4.60 & & & \\
\hline 4 & $5.08(0.000)$ & $2.55(0.000)$ & 5.11 & 3.37 & & & \\
\hline 5 & $4.53(0.000)$ & $1.57(0.000)$ & & 2.52 & & & \\
\hline 6 & $4.12(0.000)$ & $0.77(0.000)$ & & 1.86 & & & \\
\hline \multicolumn{8}{|c|}{$\mathbf{1}^{1} \mathbf{B}_{1 g}$} \\
\hline 2 & $6.54(0.000)$ & $5.26(0.000)$ & 6.85 & 5.63 & & & \\
\hline 3 & $5.38(0.000)$ & $4.20(0.000)$ & 5.75 & 4.46 & & & \\
\hline 4 & $4.41(0.000)$ & $3.35(0.000)$ & 4.82 & 3.62 & & & \\
\hline 5 & $3.68(0.000)$ & $2.70(0.000)$ & & 3.00 & & & \\
\hline 6 & $3.12(0.000)$ & $2.21(0.000)$ & & 2.44 & & & \\
\hline
\end{tabular}

with the experimental value of $4.13 \mathrm{eV}$. When going from $\mathrm{ADC}(2)$-s to $\mathrm{ADC}(2)-\mathrm{x}$, i.e. improving the description of doubly excited states to first order, its excitation energy drops by $1 \mathrm{eV}$ from 4.57 to $3.51 \mathrm{eV}$, despite its mainly single excitation character. As a consequence, $\operatorname{ADC}(2)-x$, underestimates the vertical excitation energy by $0.6 \mathrm{eV}$. Not discussing the vertical excitation energies of the $1^{1} \mathrm{~B}_{3 u}$ state of all larger linear polyacenes individually, which are listed in Table 1, an overall identical trend is observed also for the larger polyacenes.

The $1^{1} \mathrm{~B}_{2 u}\left(L_{a}\right)$ is the second lowest excited $\mathrm{S}_{2}$ state of naphthalene, exhibiting an experimental excitation energy of $4.66 \mathrm{eV}$. Also this state corresponds to a singly excited state almost exclusively represented as HOMO $\rightarrow$ LUMO excitation. $\mathrm{ADC}(2)-\mathrm{s}, \mathrm{EOM}-\mathrm{CCSD}$ and $\mathrm{CC} 2$ tend to overestimate the vertical excitation energy by $0.7-0.2 \mathrm{eV}$, while TDDFT/B3LYP underestimates it by $0.3 \mathrm{eV}$. DFT/MRCI is the only method which agrees excellently with the experimental value. Comparison of the computed $\operatorname{ADC}(2)$-s and $\operatorname{ADC}(2)$-x values of this state of 5.09 and 4.47 reveals again a large drop in excitation energy, although not as pronounced as for the $1^{1} \mathrm{~B}_{3 u}$ state above. In the case of the $1^{1} \mathrm{~B}_{2 u}$ state, the accuracy of the DFT/MRCI method degrades slightly when going to larger polyacenes, which may be related to size-consistency problems. For ADC(2)-s, EOM-CCSD, and $\mathrm{CC} 2$, the relative error in excitation energies remains essentially the same or 
improves slightly with increasing number of fused benzene rings. Astonishingly, TDDFT/B3LYP and ADC(2)-x show a very similar behavior, despite the very different nature of the methods, and give essentially identically poor values for the larger polyacenes. Both methods underestimate the excitation energy of the $1^{1} \mathrm{~B}_{2 u}$ state of hexacene by $0.5 \mathrm{eV}$. Comparison of the computed and experimental values of the $1^{1} \mathrm{~B}_{3 u}\left(L_{b}\right)$ and $1^{1} \mathrm{~B}_{2 u}\left(L_{a}\right)$ states shows that the $1^{1} \mathrm{~B}_{2 u}$ state is essentially degenerate with $1^{1} \mathrm{~B}_{3 u}$ for anthracene and becomes the lowest excited state for the larger polyacenes. This trend is correctly reproduced by all methods described above, only TDDFT/B3LYP falsely designates the $1^{1} \mathrm{~B}_{2 u}$ excited state as the lowest one already for naphthalene.

The lowest excited state with gerade symmetry and in total the third lowest excited $\mathrm{S}_{3}$ state of naphthalene is the $2^{1} \mathrm{~A}_{g}$ state at $\mathrm{ADC}(2)-\mathrm{s}, \mathrm{ADC}(2)-\mathrm{x}$ and EOMCCSD level (Table 1) exhibiting vertical excitation energies of $6.44 \mathrm{eV}, 5.04 \mathrm{eV}$ and $6.39 \mathrm{eV}$ at these levels of theory, respectively. At DFT/MRCI level, the vertical excitation energy of the $2^{1} \mathrm{~A}_{g}$ state is $5.73 \mathrm{eV}$. However, the difference can easily be due to the different ground state geometries employed within the former and the latter calculations. Nevertheless, the huge drop in excitation energy of $1.4 \mathrm{eV}$ of the $2^{1} \mathrm{~A}_{g}$ state in the transition from $\operatorname{ADC}(2)$-s to $\operatorname{ADC}(2)$-x reveals the importance of doubly excited configurations for the proper description of this state. Indeed, the $2^{1} \mathrm{~A}_{g}$ is best described as a $\mathrm{HOMO}^{2} \rightarrow \mathrm{LUMO}^{2}$ double excitation. Still, ADC(2)-x clearly underestimates the vertical excitation energy by about $0.7 \mathrm{eV}$ compared to the DFT/MRCI value. In view of the good accuracy of the DFT/MRCI approach in the description of the $1^{1} \mathrm{~B}_{3 u}$ and $1^{1} \mathrm{~B}_{2 u}$ states above, the DFT/MRCI values are certainly the most reliable ones also for the $2^{1} \mathrm{~A}_{g}$ and $1^{1} \mathrm{~B}_{1 g}$ states of the neutral polyacenes. The obtained value is also in agreement with the previously mentioned two-photon experiments on naphthalene, in which a gerade state has been located around $5.5 \mathrm{eV}$ [63-65]. Going to larger polyacenes, the accuracy of $\mathrm{ADC}(2)$-s and EOM-CCSD degrades owing to the increasing importance of doubly excited configurations in the description of the wavefunction of the $2^{1} \mathrm{~A}_{g}$ state with growing number of fused benzene rings. Remarkably, the vertical excitation energy of pentacene drops by $3 \mathrm{eV}$ going from $\mathrm{ADC}(2)$-s to $\mathrm{ADC}(2)$-x from 4.53 to $1.57 \mathrm{eV}$. However, also the ADC(2)-x and DFT/MRCI values deviate by $1 \mathrm{eV}$ for pentacene and hexacene, the $\operatorname{ADC}(2)$-x values being lower. Taking the typical 0.5 $\mathrm{eV}$ underestimation of the closed-shell $\mathrm{ADC}(2)$-x approach into account [34, 37, 41], still $0.5 \mathrm{eV}$ remain to be explained. The different ground state geometries, employed in the $\operatorname{ADC}(2)-\mathrm{x}$ and DFT/MRCI calculations can be excluded as origin of these deviations. Recalculation of the excitation energies of anthracene at the $\operatorname{ADC}(2)-x$ level using also the DFT/B3LYP/TZVP optimized molecular geometry revealed only negligible changes of less than $0.1 \mathrm{eV}$ in the vertical excitation energies. It remains to be clarified whether the observed discrepancies between DFT/MRCI and $\mathrm{ADC}(2)-\mathrm{x}$ stem from increasing multi-reference character of the ground states of the polyacenes with size which is most likely not captured by the MP2 method underlying the $\operatorname{ADC}(2)$ approach as described in Section 2.

The second lowest excited state with gerade symmetry of naphthalene is the $1^{1} \mathrm{~B}_{1 g}$ state at the levels of $\mathrm{ADC}(2)$-s, $\mathrm{ADC}(2)$-x and EOM-CCSD with excitation energies of $6.54,5.26$ and $6.85 \mathrm{eV}$, respectively. At DFT/MRCI level, as mentioned above, this state is the lowest gerade state of naphthalene exhibiting a vertical excitation energy of $5.63 \mathrm{eV}$. However, already for anthracene $1^{1} \mathrm{~B}_{1 g}$ is the second lowest gerade state also at DFT/MRCI level. As for the $2^{1} \mathrm{~A}_{g}$ state, the vertical excitation energy drops by remarkable $1.3 \mathrm{eV}$ from $\mathrm{ADC}(2)$-s to $\mathrm{ADC}(2)$-x underlining the importance of double excitations in the description of its wavefunction. In fact, the $1^{1} \mathrm{~B}_{1 g}$ state has large contributions from singly excited determinants 
Figure 2. Excitation energies of the four lowest excited states of the neutral linear polyacenes depending on the number of fused benzene rings, calculated at the $\mathrm{ADC}(2)-\mathrm{s}$ (dotted lines) and ADC(2)-x (full lines) levels using the $6-31 G^{*}$ basis set. $A_{g}$ states are given as squares $(\square), B_{1 g}$ as triangles $(\triangle), B_{2 u}$ as circles $(\odot)$ and $\mathrm{B}_{3 u}$ as stars $(*)$.

HOMO- $2 \rightarrow$ LUMO and HOMO $\rightarrow$ LUMO +2 , but with increasing size of the polyacene contributions from double excitations become more prominent. In general, the deviation between the $\mathrm{DFT} / \mathrm{MRCI}$ and $\mathrm{ADC}(2)$ - $\mathrm{x}$ values is only $0.4 \mathrm{eV}$ at most for all polyacenes, which is the typical underestimation of the $\mathrm{ADC}(2)-\mathrm{x}$ scheme.

For large molecules, it is often very difficult or even impossible to apply multireference methods to take doubly excited configurations properly into account. Thus it is very important to provide a diagnostic for the detection of states with substantial double excitation character. Comparison of the results of strict and extended $\mathrm{ADC}(2)$ calculations offers this opportunity, since the latter significantly improves the description of states with double excitation character lowering their excitation energies relative to typical single excitations. Thereby, the relevance of doubly excited configurations in the description of low-lying excited states is revealed. This has already been demonstrated for polyenes [41-43], but can also be nicely seen in Figure 2, in which the excitation energies of the four lowest excited states $1^{1} \mathrm{~B}_{3 u}, 1^{1} \mathrm{~B}_{2 u}, 2^{1} \mathrm{~A}_{g}$ and $1^{1} \mathrm{~B}_{1 g}$ obtained at $\mathrm{ADC}(2)$-s and -x level are plotted against the number of fused benzene rings. While the excitation energies of these states in general decrease from $\mathrm{ADC}(2)$-s to $\mathrm{ADC}(2)$-x by about $1 \mathrm{eV}$ shifting the curves parallel downwards, the exception is the $2^{1} \mathrm{~A}_{g}$ state. At $\mathrm{ADC}(2)$-x level the curve is much steeper than at the $\operatorname{ADC}(2)$-s level owing to the pronounced double excitation character of this state in the larger polyacenes. While the $\mathrm{ADC}(2)$-s calculations yield the $2^{1} \mathrm{~A}_{g}$ state for all polyacenes larger than naphthalene as fourth lowest excited state, it drops drastically in energy when going to the extended scheme. Indeed, the $\operatorname{ADC}(2)$-x calculations predict this state even to become the lowest excited state of pentacene and the larger polyacenes. Nevertheless, since the excitation energy of the $\mathrm{A}_{g}$ state is underestimated, the actually crossing point of 
the $2^{1} \mathrm{~A}_{g}$ and $1^{1} \mathrm{~B}_{2 u}$ most likely occurs later at hexacene, as has been predicted by the DFT/MRCI calculations. In summary, the comparison of $\mathrm{ADC}(2)$-s and $\operatorname{ADC}(2)$-x calculations is generally very helpful to identify low-lying excited states with significant double excitation character in unknown molecules for which expensive multi-reference methods are no longer feasible.

\section{Excited State Properties of Polyacene Radical Cations}

The radical cations of the linearly fused polyacenes exhibit a doublet open-shell electronic ground state, in which the positive charge is delocalized over the entire carbon backbone of the $\mathrm{D}_{2 h}$ symmetric molecules. The irreducible representation of the electronic ground state changes depending on the number of fused rings. PACs with an even number of rings exhibit a ${ }^{2} \mathrm{~A}_{u}$ ground state, while it is ${ }^{2} \mathrm{~B}_{3 g}$ for those with an odd number. However, the nature of the involved molecular orbitals stays the same for all polyacene radical cations and the frontier orbitals are displayed for naphathalene, anthracene and tetracene in Figure 3. For the classification of the molecular orbitals, we will use the following nomenclature. We will distinguish between highest doubly occupied molecular orbitals (HOMO) and lowest unoccupied molecular orbitals (LUMO), and one singly occupied molecular orbital (SOMO), since the appearance of the individually optimized $\alpha$ - and $\beta$-MOs in the unrestricted UHF reference determinant are practically identical. This is also noticed by the small spin contamination of the ground state UHF reference determinants, which is only $0.09\left(\left\langle\hat{S}^{2}\right\rangle=0.84\right)$ for the naphthalene radical cation and increases up to $0.29\left(\left\langle\hat{S}^{2}\right\rangle=1.04\right)$ for the radical cation of hexacene. The electron occupying the SOMO is assumed to exhibit $\alpha$ spin.

According to the change of the irreducible representation of the electronic ground state of the polyacene radical cations with odd or even number of fused rings, also the excited states change their symmetry classification. For example, the $\mathrm{D}_{1}$ state of the naphthalene radical cation is a ${ }^{2} \mathrm{~B}_{1 u}$ state, while the corresponding one of anthracene has ${ }^{2} \mathrm{~B}_{2 g}$ symmetry, although the nature of these states is the same for both. The symmetry of the electronic transition, however, from the ground state into the excited state is given by the direct product of the state symmetries and is for both $\mathrm{D}_{1}$ states of the radical cations of naphthalene and anthracene the same, i.e. $\mathrm{B}_{1 g}$. Therefore, the excited states of the PAC radical cations are going to be classified by their transition symmetry rather than the state symmetry. Note that for closed-shell molecules both are necessarily the same, since the ground state is always totally symmetric.

Removal of a $\beta$-electron out of the highest occupied molecular orbital of the neutral polyacenes creates a vacancy in the SOMO of the radical cations, which can be easily filled by excitation of a $\beta$-electron out of a lower lying doubly occupied orbital. Typically, these excited states exhibit very small excitation energies, sometimes even in the near IR region [81-83]. Not surprisingly, the $\mathrm{D}_{1}$ state of naphthalene corresponds in fact to such a state, which can be best represented as an excitation of an $\beta$-electron out of the HOMO into the SOMO with a transition symmetry of $\mathrm{B}_{1 g}$ (Table 2, Figure 3 ). Due to its symmetry, this transition is optically one-photon forbidden. At the theoretical levels of $\operatorname{UADC}(2)-\mathrm{s}, \operatorname{UADC}(2)-\mathrm{x}$ and UB3LYP this state exhibits vertical excitation energies for the naphthalene radical cation of as small as $1.43,0.62$ and $1.25 \mathrm{eV}$, respectively. Unfortunately, no experimental value is available for comparison. Going to larger polyacenes, the orbital ordering is slightly changed and for anthracene and tetracene the former HOMO is now the HOMO-1, which for pentacene and hexacene becomes even the 
LUMO+1

\section{Naphthalene}

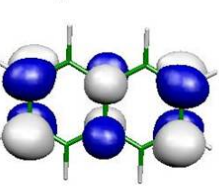

LUMO

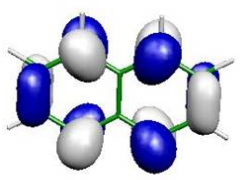

SOMO

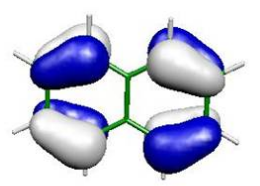

HOMO

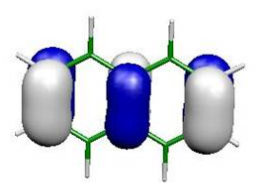

HOMO-1

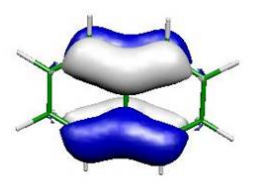

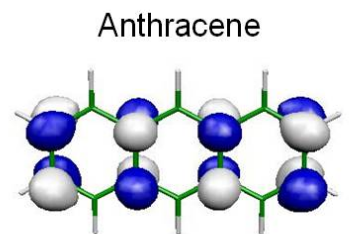
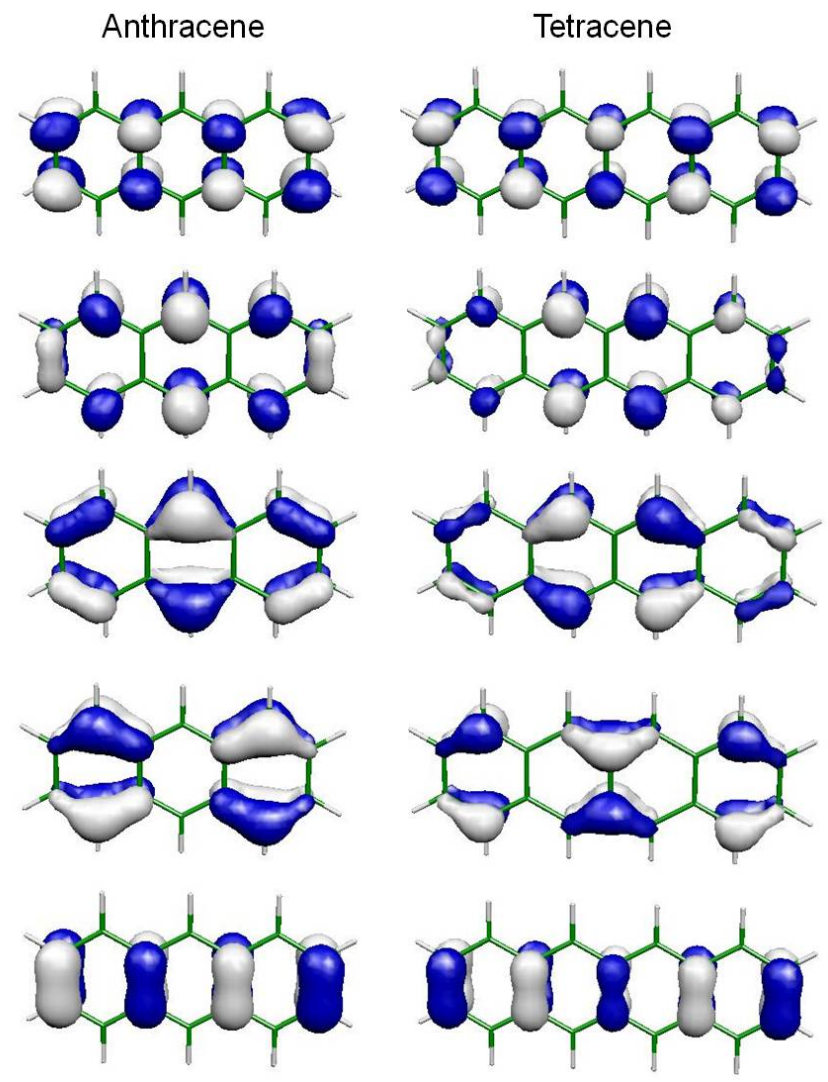

Figure 3. Frontier molecular orbitals of naphthalene, anthracene and tetracene.

HOMO-2 (Figure 3). Along with this trend, the amplitude of the $\beta$-transition out of these orbitals into the SOMO decreases from 0.89 for naphthalene to 0.50 in hexacene. Most strikingly however, the excitation energy of the $\mathrm{B}_{1 g}$ transition increases from naphthalene to hexacene to values of $2.70,1.62$ and $2.15 \mathrm{eV}$ at the $\mathrm{ADC}(2)-\mathrm{s}, \mathrm{ADC}(2)-\mathrm{x}$ and UB3LYP levels, respectively. This contradicts the typical particle-in-a-box model for excited states of molecules with extended $\pi$-systems, which predicts that the excitation energies become smaller with increasing system size. This state represents an interesting exception since the excitation energies of all other states of the investigated polyacenes do indeed drop with increasing molecular size as we will see later (Figure 4).

To understand why the $\mathrm{B}_{1 g}$ transition does behave so differently from all the other electronically excited states, one can inspect the molecular orbitals and their energies with increasing polyacene size. In figure 5, the orbital energies of the frontier orbitals are displayed. First of all, comparison of the energies of the $\alpha$ and $\beta$ orbitals reveals that the $\beta$ orbital energies are only slightly smaller than the corresponding $\alpha$ energies due to the missing energetically favorable exchange interactions in the $\beta$ space. Only the SOMO, which is occupied by an $\alpha$ electron and vacant in the $\beta$ orbital space, is shifted to substantially higher energies than the corresponding $\alpha$, since in the former it represents the energy of an additional electron. In the case of the $\mathrm{B}_{1 g}$ transition, an electron is excited from the $\beta \mathrm{HOMO}$ into the $\beta \mathrm{SOMO}$ in naphthaline, from $\beta \mathrm{HOMO}-1$ to the $\beta \mathrm{SOMO}$ in anthracene and tetracene, and from $\beta \mathrm{HOMO}-2$ to the $\beta \mathrm{SOMO}$ in the longer polyacenes, respectively. Surprisingly, the gap between these orbitals does not decrease with growing molecular size, but instead increases slightly (Figure 5). In principle, this effect can largely be ascribed to the remarkably small increase of the orbital energy of the 
Table 2. Computed vertical excitation energies $(\mathrm{eV})$ and oscillator strengths (in parenthesis) of the four lowest excited states of the radical cations of naphthalene (2) to hexacene (6) at UADC(2)-s, UADC(2)-x and UTDDFT/B3LYP of theory compared with literature data at TDDFT level and experimental values.

\begin{tabular}{l|llll}
\hline $\mathrm{n}$ & $\mathrm{UADC}(2)-\mathrm{s}$ & $\mathrm{UADC}(2)-\mathrm{x}$ & UB3LYP & UB3LYPa
\end{tabular}

\begin{tabular}{l|lll}
\multicolumn{1}{l}{$\mathbf{1 B}_{1 g}$} \\
\hline 2 & $1.43(0.000)$ & $0.62(0.000)$ & 1.25 \\
3 & $1.80(0.000)$ & $0.90(0.000)$ & 1.54 \\
4 & $2.16(0.000)$ & $1.26(0.000)$ & 1.83 \\
5 & $2.43(0.000)$ & $1.51(0.000)$ & 1.92 \\
6 & $2.70(0.000)$ & $1.62(0.000)$ & 2.15
\end{tabular}

\begin{tabular}{l|llllc}
$\mathbf{1 B}_{3 u}$ & & & \\
\hline 2 & $2.46(0.084)$ & $1.75(0.058)$ & 2.52 & 2.14 & $1.84^{\mathrm{b}, \mathrm{d}}, 1.85^{\mathrm{c}}$ \\
3 & $2.01(0.147)$ & $1.19(0.085)$ & 1.95 & 1.93 & $1.73^{\mathrm{e}}, 1.71^{\mathrm{f}}, 1.75^{\mathrm{g}}$ \\
4 & $1.78(0.221)$ & $0.99(0.121)$ & 1.72 & 1.70 & $1.43^{\mathrm{f}, \mathrm{h}, \mathrm{i}}$ \\
5 & $1.58(0.301)$ & $0.78(0.148)$ & 1.52 & 1.50 & $1.31^{\mathrm{k}}$ \\
6 & $1.40(0.385)$ & & 1.35 & 1.34 & $1.14^{\mathrm{l}}$
\end{tabular}

\begin{tabular}{l|llllc}
\multicolumn{1}{l}{$\mathbf{1 B}_{2 u}$} \\
\hline 2 & $3.68(0.013)$ & $2.74(0.012)$ & 3.43 & 2.98 & $2.72^{\mathrm{b}, \mathrm{c}}, 2.69^{\mathrm{d}}$ \\
3 & $2.61(0.014)$ & $1.79(0.008)$ & 2.38 & 2.32 & $2.02^{\mathrm{e}}$ \\
4 & $1.99(0.023)$ & $1.23(0.013)$ & 1.75 & 1.70 & $1.65^{\mathrm{f}}, 1.66^{\mathrm{h}, \mathrm{i}}$ \\
5 & $1.53(0.023)$ & $0.79(0.011)$ & 1.28 & 1.25 & $1.27^{\mathrm{k}}$ \\
6 & $1.18(0.021)$ & $0.46(0.007)$ & 0.94 & 0.90 & $0.80^{\mathrm{l}}$
\end{tabular}

\begin{tabular}{l|lll}
\multicolumn{2}{l}{$\mathbf{2 \mathbf { A } _ { g }}$} \\
\hline 2 & & & \\
\hline 3 & $3.16(0.000)$ & $2.28(0.000)$ & 3.01 \\
4 & $3.11(0.000)$ & $2.21(0.000)$ & 2.77 \\
5 & $2.85(0.000)$ & $1.98(0.000)$ & 2.50 \\
6 & $2.60(0.000)$ & & 2.24 \\
\hline
\end{tabular}

a taken from Ref. [70], who used the $6-31+\mathrm{G}^{*}$ for geometry optimization and the excited state calculations.

b taken from Ref. [71]

c taken from Ref. [72]

d taken from Ref. [73]

e taken from Ref. [74]

f taken from Ref. [75]

g taken from Ref. [76]

$\mathrm{h}$ taken from Ref. [77]

i taken from Ref. [78]

k taken from Ref. [79]

${ }^{1}$ taken from Ref. [80]

corresponding $\beta$ HOMO with increasing size of the polyacenes, which results in an essentially flat curve in Figure 5 and an increasing HOMO-SOMO gap for this electronic transition. The corresponding HOMO (naphthalene), HOMO-1 (anthracene and tetracene) or HOMO-2 (pentacene and hexacene) exhibits a characteristic non-bonding ladder-type shape (Figure 3).

Let us now return to the description of the other low-lying electronic transitions of the linear polyacene radical cations. The second excited $\mathrm{D}_{2}$ state of the naphthalene radical cation corresponds to a ${ }^{2} \mathrm{~B}_{3 u}$ transition. At the theoretical levels of $\operatorname{ADC}(2)-s, \operatorname{ADC}(2)-x$ and UB3LYP, it exhibits vertical excitation energies of 2.46, 

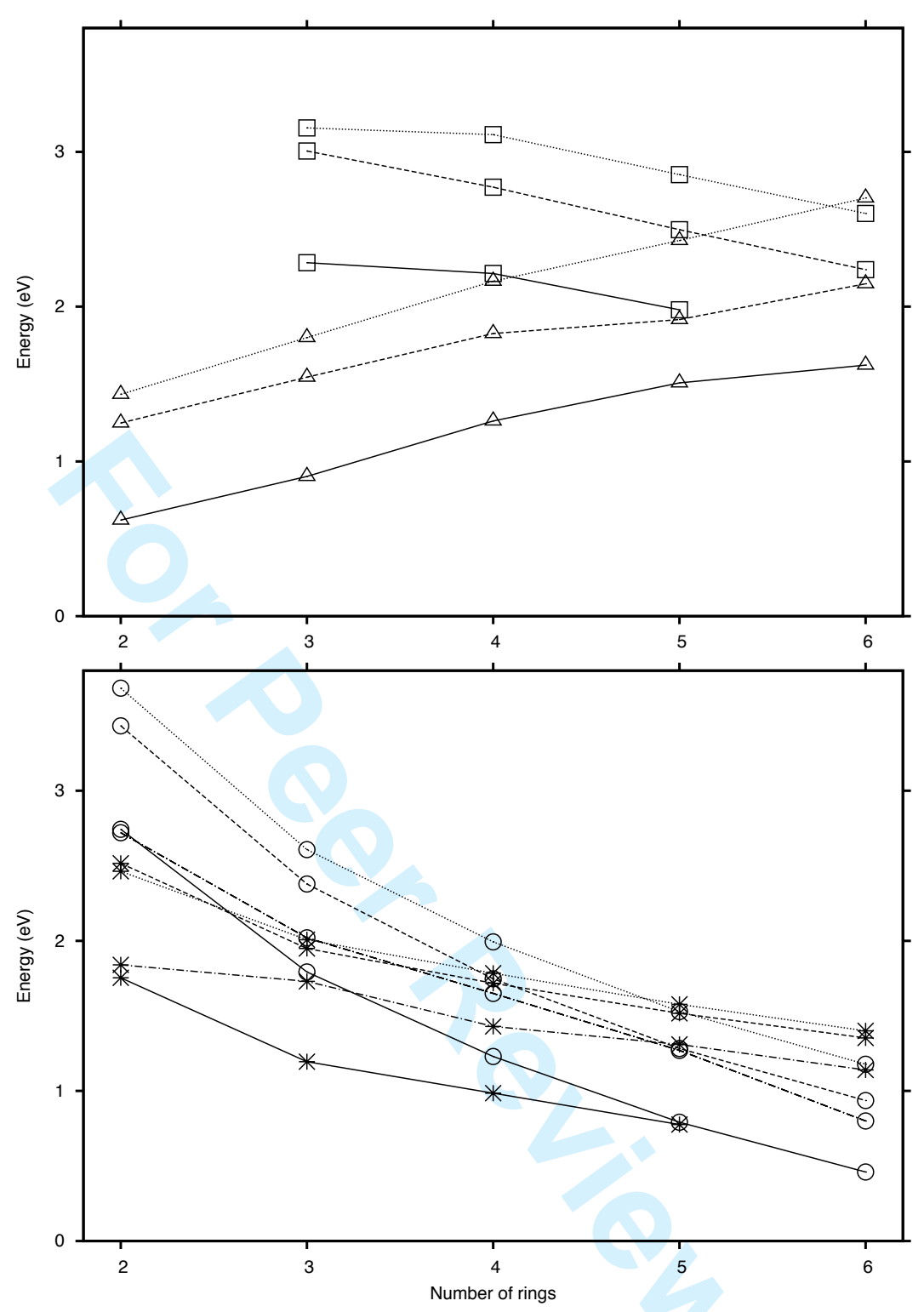

Figure 4. Comparison of the $\mathrm{A}_{g}(\square)$ and $\mathrm{B}_{1 g}(\triangle)$ states (top) and of the $\mathrm{B}_{2 u}(\odot)$ and $\mathrm{B}_{3 u}(*)$ states (bottom) of the polyacene radical cations depending on the number of fused benzene rings at the theoretical levels of UADC (2)-s (dotted line) and UADC(2)-x (full line) with UB3LYP (dashed line) and experimental values $(\cdot-)[71,74,75,79,80]$.

1.75 and $2.52 \mathrm{eV}$. Using a diffuse basis set, this transition has been calculated previously using TDDFT/B3LYP to possess an excitation energy of $2.14 \mathrm{eV}$ [70]. The experimental value is given as $1.84 \mathrm{eV}$ [71-73]. Going to the larger polyacene radical cations, tetracene for example, the vertical excitation energies amount to 1.78 , 0.99 and $1.72 \mathrm{eV}$, at the theoretical levels of $\operatorname{ADC}(2)-\mathrm{s}, \operatorname{ADC}(2)-\mathrm{x}$ and UB3LYP, respectively. Compared to the known experimental value of $1.43 \mathrm{eV}$, one recognizes the typical overestimation of the excitation energy by $\mathrm{ADC}(2)$-s and the typical underestimation by $\operatorname{ADC}(2)-\mathrm{x}$. This trend is observed also for the other excited states of the investigated polyacene radical cations. In the one-particle picture, the $\mathrm{B}_{3 u}$ transition of the naphthalene radical cation is best described as a transition of 

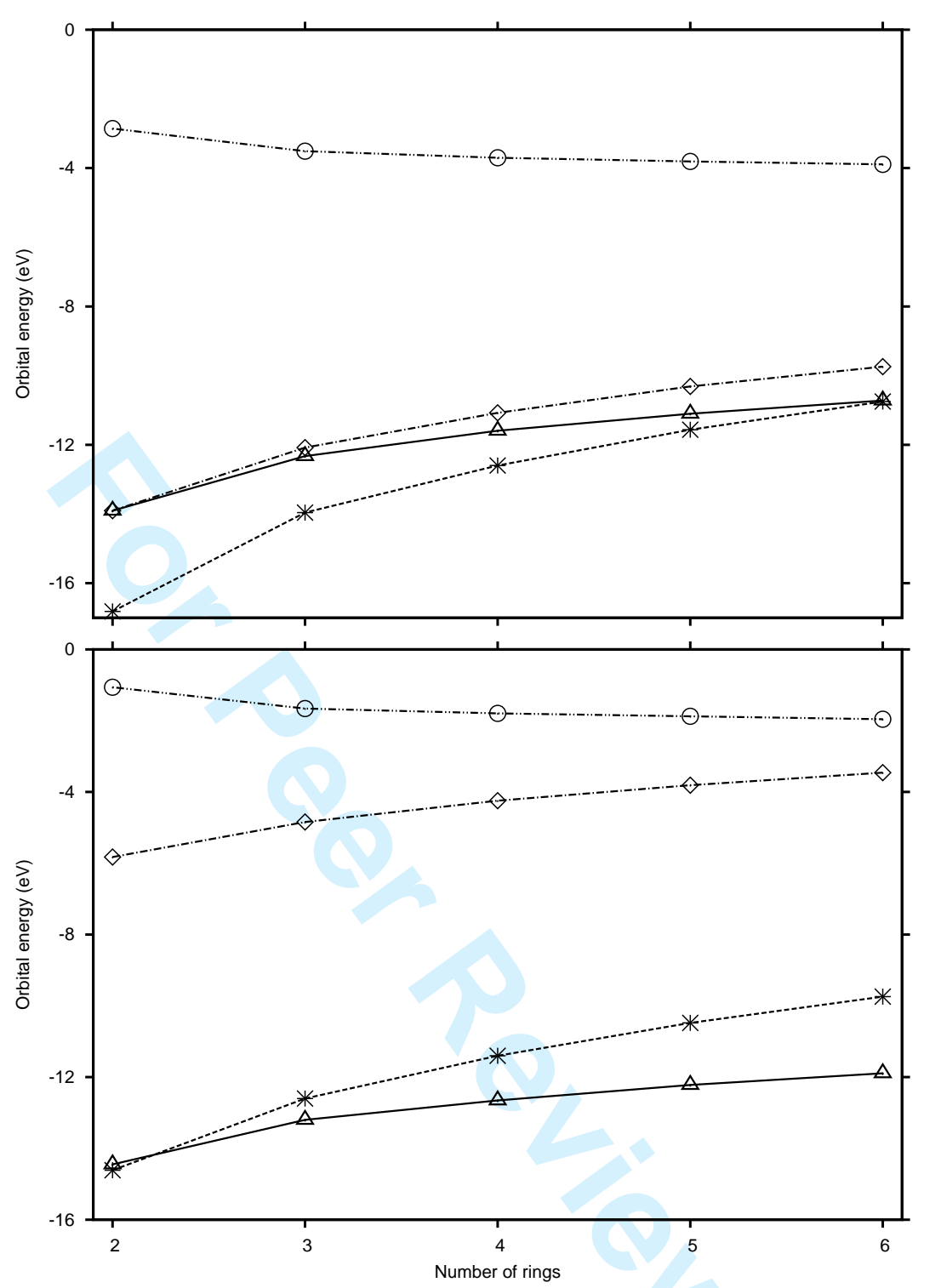

Figure 5. Orbital energies of the alpha (top) and beta (bottom) orbitals of the polyacenes as a function of the number of fused benzene rings. (HOMO, full line, $\triangle$; HOMO-1, --, *; SOMO,,$- \diamond ; \mathrm{LUMO}, \cdots-$, $\odot$ ).

a $\beta$ electron from the HOMO-1 into the SOMO. For the larger polyacene cations the HOMO and HOMO-1 orbitals change and then the $\mathrm{B}_{3 u}$ transition becomes the HOMO to SOMO excitation (Figure 3). However, the weight of the corresponding determinant in the wavefunctions is almost constant for all polyacene radical cations with 0.87 from naphthalene to 0.82 for hexacene. Since the excitation energy of the $\mathrm{B}_{3 u}$ transition decreases with increasing system size, it becomes the lowest excited state for tetracene at $\operatorname{ADC}(2)$ level, in agreement with the experimental data $[75,77,78]$. Since the $\mathrm{B}_{3 u}$ transition is ungerade, it is one-photon allowed and thus exhibits substantial oscillator strength increasing from naphthalene with values of 0.084 and 0.058 at the theoretical levels of $\mathrm{ADC}(2)$-s and $\mathrm{ADC}(2)$-x, respectively, to 0.301 and 0.148 for pentacene, for example. This transition can be expected to dominate the absorption spectra of the polyacene radical cations in 
the low-energy region.

The third lowest excited $\mathrm{D}_{3}$ state of the naphthalene radical cation corresponds to an one-photon allowed $\mathrm{B}_{2 u}$ transition exhibiting a vertical excitation energy of 3.68, 2.74 and $3.43 \mathrm{eV}$ at the theoretical levels of $\mathrm{ADC}(2)-\mathrm{s}, \mathrm{ADC}(2)-\mathrm{x}$ and UB3LYP, respectively. The experimental excitation energy has been found to be about 2.7 $\mathrm{eV}$ [71-73] for the $\mathrm{B}_{2 u}$ transition of the naphthalene radical cation, which is in good agreement with the $\mathrm{ADC}(2)$-x value. For the longer polyacene radical cations, however, $\operatorname{ADC}(2)$-x underestimates the experimental values by about $0.2-0.4 \mathrm{eV}$ (Table 2). Going to larger polyacene radical cations, the $\mathrm{B}_{2 u}$ transition crosses the $\mathrm{B}_{3 u}$ transition and also the $\mathrm{B}_{1 g}$ transition and eventually becomes the lowest one for pentacene (Table 2, Figure 4). In the molecular orbital picture, the $\mathrm{B}_{2 u}$ electronic transition is best described as a linear combination of two determinants, one corresponding to an excitation of an $\alpha$ electron from the SOMO to the LUMO (Figure 3 ) and one corresponding to a $\beta$-electron excitation out of the HOMO-2 into the SOMO. While the latter dominates the transition for the smaller polyacenes, the first dominates for the longer ones exhibiting transition amplitudes of about 0.8 from tetracene on. The oscillator strength of the $1 \mathrm{~B}_{2 u}$ transition is overall rather small at the ADC levels (Table 2).

A gerade $\mathrm{A}_{g}$ transition is the fourth lowest excited $\mathrm{D}_{4}$ state of the radical cations of anthracene to pentacene, and the third lowest one of the hexacene radical cation, since it crosses the $\mathrm{B}_{1 g}$ transition. For the naphthalene radical cation an analoguous excited state does not exist. In the one-particle picture, the $\mathrm{A}_{g}$ transition is best described as an excitation of a $\beta$-electron from the HOMO-2 to the SOMO in anthracene and tetracene or, since HOMO-2 and HOMO-1 change when going to pentacene and hexacene, from the HOMO-2 into the SOMO in the latter two longer polyacenes. The weight of the corresponding determinants in the excited state wavefunctions of the $\mathrm{D}_{4}$ state, range between 0.78 and 0.85 for all polyacene radical cations. Due to its symmetry, the $\mathrm{A}_{g}$ transition is strictly one-photon forbidden, and cannot directly be observed in corresponding experimental spectra. At the level of $\mathrm{ADC}(2)$-s the $\mathrm{A}_{g}$ transition exhibits excitation energies of 3.15, 3.11, 2.85 and $2.6 \mathrm{eV}$ from anthracene up to hexacene, i.e. the vertical excitation energies decrease only very slowly with increasing polyacene size (Figure 4). Going to the $\operatorname{ADC}(2)-x$ level, the excitation energies drop consistently by about $0.9 \mathrm{eV}$ to 2.28 , 2.21 and $1.98 \mathrm{eV}$ for anthracene, tetracene and pentacene, respectively. Since this state does not exhibit substantial contributions of doubly excited configurations, also unrestricted TDDFT can describe this state reasonably well. The excitation energies are found to lie between the ones given by $\operatorname{ADC}(2)-\mathrm{s}$ and $\operatorname{ADC}(2)-\mathrm{x}$ and exhibit values of $3.01,2.77,2.50$ and $2.24 \mathrm{eV}$ for the polyacenes from anthracene to hexacene, respectively.

Let us briefly comment on the performance of the UADC schemes and UTDDFT for the vertical excitation energies of the polyacene radical cations. It has been observed previously that $\mathrm{ADC}(2)$-s tends to overestimate excitation energies by $0.3-0.5 \mathrm{eV}$, while $\mathrm{ADC}(2)$-x usually underestimates them by about $0.3 \mathrm{eV}$ [42]. This is also observed here for the electronic transitions for which experimental data are available for comparison, i. e. $\mathrm{B}_{2 u}$ and $\mathrm{B}_{3 u}$ : $\mathrm{ADC}(2)$-s yields too large values by about 0.3 to $0.7 \mathrm{eV}$, while $\mathrm{ADC}(2)$-x gives too small values. Unrestricted TDDFT employing the B3LYP xc-functional and the $6-31 \mathrm{G}^{*}$ basis set again overestimates the excitation energies by about by 0.2 to $0.3 \mathrm{eV}$ and gives the best agreement with the available experimental data. However, this is true only for the one-photon allowed ungerade $\mathrm{B}_{2 u}$ and $\mathrm{B}_{3 u}$ transitions, for which doubly excited configurations do not play an important role. The accuracy of the gerade transitions $\mathrm{B}_{1 g}$ and $\mathrm{A}_{g}$ is difficult to estimate, since neither experimental nor theoretical benchmark data 


\section{Brief Summary}

The energetically low-lying excited electronic states of linearly fused polyacenes and their radical cations have been investigated using the algebraic diagrammatic construction (ADC) scheme of second order $(\operatorname{ADC}(2))$. The neutral polyacenes generally pose a challenge to electronic structure theory due to an increasing multi-reference character of the ground state with increasing molecular size and the importance of doubly excited configurations for their excited states with gerade spatial symmetry. In addition, the availability of reliable multi-reference data, makes the polyacenes an ideal test set to challenge the accuracy and applicability of single-reference $\operatorname{ADC}(2)$ for such problematic cases. Our calculations have shown that strict $\mathrm{ADC}(2)(\mathrm{ADC}(2)-\mathrm{s})$ can reproduce the trends of the singly excited states $\mathrm{B}_{2 u}$ and $\mathrm{B}_{3 u}$ with essentially the same accuracy as CC2, and, analogously, can not describe the gerade states $\mathrm{A}_{g}$ and $\mathrm{B}_{1 g}$ exhibiting mostly double excitation character. The description of these doubly excited states is largely improved when extended $\mathrm{ADC}(2)(\mathrm{ADC}(2)-\mathrm{x})$ is used, and the excitation energies are observed to drop substantially from the $\operatorname{ADC}(2)$-s to the $\operatorname{ADC}(2)$-x level. However, for the longer polyenes an atypically large underestimation of the excitation energies at $\mathrm{ADC}(2)-\mathrm{x}$ level is observed, which is attributed to the pronounced multi-reference character of the neutral polyacenes. Nevertheless, a combined application of both $\operatorname{ADC}(2)$-s and $\operatorname{ADC}(2)-x$ is extremely useful for the identification of the importance of doubly excited configuration for a correct description of the excited state spectrum of unknown molecules, for which expensive multi-reference approaches are not feasible.

The theoretical investigation of the radical cations of the polyacenes employing unrestricted ADC (UADC) revealed that neither doubly excited states nor a multireference treatment are critically important for the theoretical description of the low-lying excited states. While $\operatorname{UADC}(2)$-s tends to overestimate the excitation energies by about $0.3-0.5 \mathrm{eV}, \mathrm{UADC}(2)-\mathrm{x}$ yields too low excitation energies by essentially the same amount. Also UTDDFT is capable of describing the low-lying excited states of the polyacenes reasonably well.

As is generally expected, the vertical excitation energies of the neutral polyacenes have been found to decrease with increasing molecular size. This is also the case for most of the excited states of the radical cations, however, our computations identified one $\mathrm{B}_{1 g}$ transition, whose excitation energy increases with increasing size of the polyacenes. This excited state can be described as an excitation of a $\beta$ electron out of the lowest doubly occupied molecular orbital (HOMO) into the singly occupied one (SOMO). Such states do not exist in neutral closed-shell systems, and are here made possible by ionization, i.e. removal of an electron out of the HOMO and thereby creating a vacancy into which another electron can now be excited. The fact that the excitation energy of this transition increases with increasing polyacene size is explained with the increasing energy gap between the orbital out of which is excited and the SOMO. 


\section{Acknowledgements}

AD acknowledges financial support from the Deutsche Forschungsgemeinschaft as a Heisenberg professor. Computation time has been generously provided by the Center of Scientific Computation of the University of Frankfurt.

\section{References}

[1]S. Grimme, Rev. Comp. Chem. 20, 153 (2004).

[2] A. Dreuw, Chem. Phys. Chem. 7, 2259 (2006).

[3]E. Runge and E.K.U. Gross, Phys. Rev. Lett. 52, 997 (1984).

[4] M.E. Casida, in , edited by D. P. Chong (World Scientific, Singapore, 1995), pp. 155-192.

[5]A. Dreuw and M. Head-Gordon, Chem. Rev. 105, 4009 (2005).

[6]D. Hegarthy and M.A. Robb, Mol. Phys. 38, 1795 (1979).

[7]R.H.E. Eade and M.A. Robb, unknownChem. Phys. Lett. 83, 362 (1981).

[8] H. Nakatsuji and K. Hirao, J. Chem. Phys. 68, 2053 (1978).

[9] O. Christiansen, H. Koch and P. Jørgensen, J. Chem. Phys. 103, 7429 (1995).

[10] O. Christiansen, H. Koch and P. Jørgensen, Chem. Phys. Lett. 243, 409 (1995).

[11]H. Koch, O. Christiansen, P. Jørgensen, T. Helgaker and A.S. de Meras, J. Chem. Phys. 106, 1808 (1997).

[12] C. Haettig and F. Weigend, J. Chem. Phys. 113, 5154 (2000).

[13] A.L. Sobolewski and W. Domcke, Eur. Phys. J. D 20, 369 (2002).

[14] A.L. Sobolewski, W. Domcke, C. Dedonder-Lardeux and C. Jouvet, Phys. Chem. Chem. Phys. 4, 1093 (2002).

[15] A.L. Sobolewski and W. Domcke, Phys. Chem. Chem. Phys. 6, 2763 (2004).

[16]S. Grimme and M. Waletzke, J. Chem. Phys. 111, 5645 (1999).

[17]K.A. Seefeld, C. Plützer, D. Löwenich, T. Häber, L. R, K. Kleinermanns, J. Tatchen and C.M. Marian, Phys. Chem. Chem. Phys. 7, 3021 (2005).

[18] C.M. Marian, D. Nolting and R. Weinkauf, Phys. Chem. Chem. Phys. 7, 3306 (2005).

[19]A. Damjanovic, H.M. Vaswani, P. Fromme and G.R. Fleming, J. Phys. Chem. B 106, 10251 (2002).

[20]M. Dierksen and S. Grimme, J. Chem. Phys. 120, 3544 (2004).

[21]M. Dierksen and S. Grimme, J. Phys. Chem. A 108, 10225 (2004).

[22] M. Garavelli, p. Celani, F. Bernardi, M.A. Robb and M. Olivucci, J. Am. Chem. Soc. 119, 6891 (1997).

[23] A. Migani, M.A. Robb and M. Olivucci, J. Am. Chem. Soc. 125, 2804 (2003).

[24]F. Blomgren and S. Larsson, Chem. Phys. Lett. 376, 704 (2003).

25]K. Emrich, Nuc. Phys. A351, 379 (1981).

[26]H. Sekino and R.J. Bartlett, Int. J. Quant. Chem. Symp. 18, 255 (1984).

[27]H.J. Monkhorst, Int. J. Quant. Chem. Symp. 11, 421 (1977).

[28]D. Mukherjee and P. Mukherjee, Chem. Phys. 39, 325 (1979).

[29] H. Koch and P. Jørgensen, J. Chem. Phys. 93, 3333 (1990).

[30]P.E.M. Siegbahn, Chem. Phys. Lett. 109, 417 (1984).

[31]B.O. Roos, Adv. Chem. Phys. 69, 399 (1987).

[32] M.A. Robb and U. Niazi, Reports in Molecular Theory 1, 23 (1990).

[33] J. Schirmer, Phys. Rev. A 26, 2395 (1982).

[34]A.B. Trofimov and J. Schirmer, J. Phys. B 28, 2299 (1995).

[35] A.B. Trofimov, G. Stelter and J. Schirmer, J. Chem. Phys. 111, 9982 (1999).

[36] A.B. Trofimov, G. Stelter and J. Schirmer, J. Chem. Phys. 117, 6402 (2002).

[37] J. Schirmer and A.B. Trofimov, J. Chem. Phys. 120, 11449 (2004).

[38] J. Linderberg and Y. Öhrn, Propagators in Quantum Chemistry (, , 2004).

[39] L.S. Cederbaum, in Encyclopedia of Computational Chemistry, edited by P. v. R. Schleyer, N. L. Clark, J. Gasteiger, H. F. Schaefer III. and P. R. Schreiner (Wiley, Chichester, 1998).

[40]C. Haettig, Adv. Quant. Chem. 50, 37 (2005).

[41] J.H. Starcke, M. Wormit, J. Schirmer and A. Dreuw, Chem. Phys. 329, 39 (2006).

[42] J.H. Starcke, M. Wormit and A. Dreuw, J. Chem. Phys. 130, 024104 (2009).

[43] J.H. Starcke, M. Wormit and A. Dreuw, J. Chem. Phys. 131, 144311 (2009).

[44]R.F. Curl, Nature 363, 14 (1993).

[45] C.J. Pope, J.A. Marr and J.B. Howard, J. Chem. Phys. 97, 11001 (1993).

[46]A.G.G.M. Tielens, The Physics and Chemistry of the Interstellar Medium (Cambridge University Press, Cambridge, 2005).

[47]D. Biermann and W. Schmidt, J. Am. Chem. Soc. 102, 3163 (1986).

[48] K.N. Houk, P.S. Lee and M.J. Nendel, J. Org. Chem. 66, 5571 (2001).

[49] V.Y. Butko, X. Chi, D.V. Lang and A.P. Ramirez, Appl. Phys. Lett. 83, 4773 (2003).

[50]J. Lee, S.S. Kim, K. Kim, J.H. Kim and S. Im, Appl. Phys. Lett. 84, 1701 (2004).

[51] M. Bendikov, F. Wudl and D.F. Perepichka, Chem. Rev. 104, 4891 (2004).

[52] M. Bendikov, H.M. Duong, K. Starkey, K.N. Houk, E.A. Carter and F. Wudl, J. Am. Chem. Soc. 126, 7416 (2004).

[53] E.S. Kadantsev, M.J. Stott and A. Rubio, J. Chem. Phys. 124, 134901 (2006).

[54] F. Mertins and J. Schirmer, Phys. Rev. A 53, 2140 (1996).

[55]R. Manne, Chem. Phys. Lett. 45, 470 (1977).

[56]E. Dalgaard, Int. J. Quant. Chem. 15, 169 (1979).

[57] J. Schirmer, Phys. Rev. A 43, 4647 (1991). 
[58] J. Schirmer and F. Mertins, Int. J. Quant. Chem. 58, 329 (1996).

[59]F. Mertins, J. Schirmer and A. Tarantelli, Phys. Rev. A 53, 2153 (1996).

[60] J. Schirmer and F. Mertins, Theo. Chem. Acc. 125, 145 (2010).

[61]Y. Shao, L.F. Molnar, Y. Jung, J. Kussmann, C. Ochsenfeld, S.T. Brown, A.T.B. Gilbert, L.V. Slipchenko, S.V. Levchenko, D.P. O'Neill, R.A.D. Jr., R.C. Lochan, T. Wang, G.J.O. Beran, N.A. Besley, J.M. Herbert, C.Y. Lin, T.V. Voorhis, S.H. Chien, A. Sodt, R.P. Steele, V.A. Rassolov, P.E. Maslen, P.P. Korambath, R.D. Adamson, B. Austin, J. Baker, E.F.C. Byrd, H. Dachsel, R.J. Doerksen, A. Dreuw, B.D. Dunietz, A.D. Dutoi, T.R. Furlani, S.R. Gwaltney, A. Heyden, S. Hirata, C.P. Hsu, G. Kedziora, R.Z. Khalliulin, P. Klunzinger, A.M. Lee, M.S. Lee, W. Liang, I. Lotan, N. Nair, B. Peters, E.I. Proynov, P.A. Pieniazek, Y.M. Rhee, J. Ritchie, E. Rosta, C.D. Sherrill, A.C. Simmonett, J.E. Subotnik, H.L.W. III, W. Zhang, A.T. Bell and A.K. Chakraborty, Phys. Chem. Chem. Phys. 8, 3172-3191 (2006).

[62] A.I. Krylov, Ann. Rev. Phys. Chem. 59, 433 (2008).

[63] A. Bergmann and J. Jortner, Chem. Phys. Lett. 26, 323 (1974).

[64] N. Mikami and M. Ito, Chem. Phys. Lett. 31, 472 (1975).

[65]R.T. Lynch, Jr. and H. Lotem, J. Chem. Phys. 66, 1905 (1977).

[66]P. Tavan and K. Schulten, J. Chem Phys. 70, 5415 (1979).

[67]C.M. Marian and N. Gilka, J. Chem. Theo. Comp. 4, 1501 (2008).

[68]S. Grimme and M. Parac, Chem.Phys.Chem. 4, 292 (2003).

69] M. Parac and S. Grimme, Chem. Phys. 292, 11 (2003).

[70] G. Malloci, G. Mulas, G. Cappellini and C. Joblin, Chem .Phys. 340, 43 (2007).

[71]F. Salama and L.J. Allamandola, J. Chem. Phys. 94, 6964 (1991).

[72] T. Pino, N. Boudin and P. Brechignac, J. Chem. Phys. 111, 7337 (1999).

[73]B.J. Kelsall and L. Andrews, J. Chem. Phys. 76, 5005 (1982).

[74] J. Szczepanski, M. Vala, D. Talbi, O. Parisel and Y. Ellinger, J. Chem. Phys. 98, 4494 (1993).

[75]T. Shida and S. Iwata, J. Am. Chem. Soc. 95, 3473 (1973).

[76] O. Sukhorukov, A. Staicy, E. Diegel, G. Rouille, T. Henning and F. Huisken, Chem. Phys. Lett. 386, 259 (2004).

[77]L. Andrews, R.S. Friedmann and B.J. Kelsall, J. Phys. Chem. 89, 4016 (1985).

[78] J. Szczepanski, J. Drawdy, C. Walburg and M. Vala, Chem. Phys. Lett. 245, 539 (1995).

[79] T.M. Halasinski, D.M. Hudgins, F. Salama, L.J. Allamanola and T. Bally, J. Phys. Chem. A 104, 7484 (2000).

[80]R. Mondal, C. Tönshoff, D. Khon, D.C. Neckers and H.F. Bettinger, J. am. Chem. Soc. 131, 14281 (2009).

[81]T.M. Halasinski, J.L. Weisman, R. Ruiterkamp, T.J. Lee, F. Salama and M. Head-Gordon, J. Phys. Chem. A 107, 3660 (2003).

[82] J.L. Weisman, T. J.Lee, F. Salama and M. Head-Gordon, Astrophys. J. 587, 256 (2003).

[83] J.L. Weisman, A. Mattioda, T. J.Lee, D. Hudgins, L.J. Allamandola, C.W. Bauschlicher and M. HeadGordon, Phys. Chem. Chem. Phys. 7, 109 (2005). 